\title{
Inferring the dense nuclear matter equation of state with neutron star tides
}

\author{
Pantelis Pnigouras ${ }^{1,2, *}$, Nils Andersson ${ }^{3}$, and Andrea Passamonti ${ }^{4}$ \\ ${ }^{1}$ Dipartimento di Fisica, "Sapienza" Università di Roma \& Sezione INFN Roma1, Piazzale Aldo Moro \\ 2, 00185 Roma, Italy \\ ${ }^{2}$ Department of Physics, Aristotle University of Thessaloniki, 54124 Thessaloniki, Greece \\ ${ }^{3}$ School of Mathematics and STAG Research Centre, University of Southampton, Southampton SO17 \\ 1BJ, UK \\ ${ }^{4}$ Via Greve 10, 00146 Roma, Italy
}

\begin{abstract}
During the late stages of a neutron star binary inspiral finite-size effects come into play, with the tidal deformability of the supranuclear density matter leaving an imprint on the gravitational-wave signal. As demonstrated in the case of GW170817 - the first direct detection of gravitational waves from a neutron star binary-this can lead to strong constraints on the neutron star equation of state. As detectors become more sensitive, effects which may have a smaller influence on the neutron star tidal deformability need to be taken into consideration. Dynamical effects, such as oscillation mode resonances triggered by the orbital motion, have been shown to contribute to the tidal deformability, especially close to the neutron star coalesence, where current detectors are most sensitive. We calculate the contribution of the various stellar oscillation modes to the tidal deformability and demonstrate the (anticipated) dominance of the fundamental mode. We show what the impact of the matter composition is on the tidal deformability, as well as the changes induced by more realistic additions to the problem, e.g. the presence of an elastic crust. Finally, based on this formulation, we develop a simple phenomenological model describing the effective tidal deformability of neutron stars and show that it provides a surprisingly accurate representation of the dynamical tide close to merger.
\end{abstract}

\section{Introduction}

The breakthrough detections of gravitational waves from binary neutron star inspirals [1,2] have led to renewed focus on the elusive neutron star equation of state. The problem has a number of complicating aspects-both relating to the observational data and the theoretical underpinning - but the essential question is quite simple: to what extent can we use observations to constrain the state and composition of matter under the extreme conditions that neutron stars represent?

Much of the recent focus has been on the neutron star tidal deformability, essentially the extent to which the tidal interaction with a binary companion deforms the neutron star fluid. This is a useful measure as it can be extracted from (or, at least, constrained by) the gravitational-wave signal [3, 4]. Notably, the celebrated GW170817 event [1] has led to a

\footnotetext{
*e-mail: pantelis.pnigouras@uniroma1.it
} 
constraint on a suitable weighted average tidal deformability, corresponding (roughly) to a neutron star radius in the range $10-13 \mathrm{~km} \mathrm{[5]} \mathrm{(the} \mathrm{result} \mathrm{is} \mathrm{somewhat} \mathrm{model} \mathrm{dependent).}$ Moreover, as this radius range agrees well with the constraints obtained from the "Neutron Star Interior Composition Explorer" (NICER) observations of PSR J0030+0451 [6, 7] and PSR J0740+6620 [8, 9], a consistent picture is beginning to emerge.

The deformability (often expressed in terms of the dimensionless Love number, $k_{l}$ ) represents the static contribution to the neutron star's tidal response (equilibrium tide). In addition, there is a dynamical tide. This is traditionally represented by the excitation of the different oscillation modes of the star. The resonance problem was first considered some time ago [1012 ], but the issue is back in focus following the suggestion that the (fundamental) $f$-mode of the star may be excited to a relevant level, even though it may not reach resonance during the inspiral [13-15]. The associated effect on the inspiral signal is weak, but its inclusion has been demonstrated to improve waveform models [16, 17].

An interesting question to pose is to what extent the composition of the neutron star matter enters the problem. As the star is deformed by the tidal interaction, matter is driven out of equilibrium and it is easy to argue that the relevant nuclear reactions are too slow to re-establish equilibrium on the time scale of inspiral. For neutron star cores dominated by a conglomerate of neutrons, protons, and electrons, the relevant equilibration time scales are

$$
t_{m U} \sim \frac{2 \text { months }}{T_{9}^{6}}, \quad t_{d U} \sim \frac{20 \mathrm{~s}}{T_{9}^{4}},
$$

for the modified and direct Urca reactions, respectively. The temperature is scaled to hot systems, $T_{9} \equiv T / 10^{9} \mathrm{~K}$, but inspiraling neutron stars are old and cold, typically in the range $T_{9} \leq 0.01$, which would make both $t_{m U}$ and $t_{d U}$ much longer than the time it takes a given system to move through the sensitivity band of a ground-based interferometer ( $\sim$ minutes).

The upshot of the equilibration argument is that the equation of state is no longer barotropic, as has been assumed in virtually every previous analysis of the tidal problem. Hence, we want to establish to what extent a "frozen" matter composition leads to a noticeable effect on, for example, the Love number, and whether this in turn affects the extraction of neutron star parameters from an observed signal.

In the following, we outline the work presented in Refs. $[15,18,19]$.

\section{The effective Love number}

The tide raised by a binary companion (here treated as a point particle) induces a linear response in the primary. In order to quantify this response, we solve the linearised fluid equations in Newtonian gravity. Assuming that the star is non-rotating, the tidal perturbation is described by the Euler equation

$$
-\omega^{2} \xi_{i}+\frac{1}{\rho} \nabla_{i} \delta p-\frac{1}{\rho^{2}} \delta \rho \nabla_{i} p+\nabla_{i} \delta \Phi=-\nabla_{i} \chi
$$

where $\xi_{i}$ denotes the tidal displacement vector, $\rho$ is the density, $p$ is the pressure, $\Phi$ is the gravitational potential, $\delta$ denotes Eulerian perturbations, and $\omega$ is the tidal frequency. In a coordinate system centred on the primary, which we will take to have mass $M_{\star}$, the tidal potential $\chi$ is given by

$$
\chi=-G M^{\prime} \sum_{l \geq 2} \sum_{m=-l}^{l} \frac{W_{l m} r^{l}}{D^{l+1}(t)} Y_{l m} e^{-i m \psi(t)},
$$

where $M^{\prime}$ is the mass of the secondary. The orbit of the companion is taken to be in the plane $[D(t), \pi / 2, \psi(t)]$ where $D$ is the binary separation and $\psi$ is the orbital phase. For $l=$ 
2 (which leads to the main contribution to the gravitational-wave signal), we have $W_{20}=$ $-\sqrt{\pi / 5}, W_{2 \pm 2}=\sqrt{3 \pi / 10}$, and $W_{2 \pm 1}=0$.

We now aim to express the driven response of the fluid in terms of a set of normal modes, corresponding to solutions $\xi_{n}$ (where $n$ is a label that identifies the modes, say in terms of the number of nodes in the radial eigenfunction and the corresponding spherical harmonics). Letting the mode frequency be $\omega_{n}$, we have

$$
\xi^{i}=\sum_{n} a_{n} \xi_{n}^{i}
$$

The Love number is generally defined as

$$
k_{l}=\frac{1}{2} \frac{\delta \Phi(R)}{\chi(R)}
$$

Then, after expressing the perturbed gravitational potential in terms of the displacement vector, we get

$$
k_{l}=-\frac{1}{2}+\frac{2 \pi}{2 l+1} \sum_{n} \frac{\tilde{Q}_{n}^{2}}{\tilde{\omega}_{n}^{2}-\tilde{\omega}^{2}}\left[1-\tilde{\omega}^{2}\left(\frac{V_{n}}{W_{n}}\right)_{R}\right]\left[1-\tilde{\omega}_{n}^{2}\left(\frac{V_{n}}{W_{n}}\right)_{R}\right]^{-1}
$$

where frequencies have been normalised as $\tilde{\omega}=\omega /\left(G M_{\star} / R^{3}\right)$, each mode is decomposed into its radial and horizontal components as

$$
\xi_{n}^{i}=\left(W_{n} \frac{\nabla^{i} r}{r}\right) Y_{l m}+V_{n} \nabla^{i} Y_{l m}
$$

and we have introduced the "overlap integral"

$$
\tilde{Q}_{n}=-\frac{1}{M_{\star} R^{l}} \int \delta \rho_{n}^{*} r^{l+2} \mathrm{~d} r
$$

In the low-frequency limit (i.e., in the equilibrium tide approximation), we get

$$
k_{l} \approx-\frac{1}{2}+\frac{2 \pi}{2 l+1} \sum_{n} \frac{\tilde{Q}_{n}^{2}}{\tilde{\omega}_{n}^{2}}\left[1-\tilde{\omega}_{n}^{2}\left(\frac{V_{n}}{W_{n}}\right)_{R}\right]^{-1} .
$$

As a quantitative test of Eq. (9), we compare results for three models corresponding to a background configuration with a polytropic equation of state of the form $p \propto \rho^{\Gamma}$, with $\Gamma=2$. Since, as noted before, the nuclear reactions required to establish chemical equilibrium are too slow to act on the inspiral timescale, it would be reasonable to assume that the composition of a perturbed fluid element is held frozen, as the system sweeps through the sensitivity band of a ground based detector. This changes the response of the stellar fluid to the tidal driving which, in turn, allows us to estimate the impact the matter composition has on the problem. Composition gradients can be accounted for by adjusting the adiabatic index $\Gamma_{1}=$ $(\partial \ln p / \partial \ln \rho)_{\mathrm{ad}}$, which is evaluated at fixed composition. Our reference model is barotropic $\left(\Gamma_{1}=\Gamma\right)$ and we compare it to two stratified models, with $\Gamma_{1}=2.05$ and $7 / 3$, respectively.

The numerical results, listed in Table 1 , demonstrate the relative importance of $g$-modes (buoyancy modes) for strongly stratified models. In each case, the mode sum converges to the expected value for the Love number which, for a barotropic model with $\Gamma=2$, should be $k_{l} \approx$ 0.259909 and the results from Table 1 do, indeed, converge towards this number. This tells us that the sum over the star's different oscillation modes provides an alternative representation 
Table 1. The accumulated contribution to the Love number from the different modes, in order of relevance of the contribution, for the three different models we consider. We expect (for the barotropic $\Gamma=2$ case) to have $k_{l} \approx 0.259909$. If we add up the contributions from the different modes in each case, the result converges to the expected answer. The mode sum is always dominated by the $f$-mode, with a modest correction from the other modes of the star, but the enhanced importance of the $g$-modes with increasing stratification is notable.

\begin{tabular}{|cc|cc|cc|}
\multicolumn{2}{c}{$\Gamma_{1}=2$} & \multicolumn{2}{c}{$\Gamma_{1}=2.05$} & \multicolumn{2}{c}{$\Gamma_{1}=7 / 3$} \\
mode & $k_{l}$ & mode & $k_{l}$ & mode & $k_{l}$ \\
\hline$f$ & 0.27528 & $f$ & 0.27055 & $f$ & 0.24685 \\
$+p_{1}$ & 0.25887 & $+p_{1}$ & 0.25526 & $+g_{1}$ & 0.26115 \\
$+p_{2}$ & 0.26021 & $+p_{2}$ & 0.25653 & $+p_{1}$ & 0.25052 \\
$+p_{3}$ & 0.26015 & $+g_{1}$ & 0.25878 & $+g_{2}$ & 0.25556 \\
& & $+g_{2}$ & 0.25960 & $+p_{2}$ & 0.25653 \\
& & $+g_{3}$ & 0.25993 & $+g_{3}$ & 0.25856 \\
& & $+g_{4}$ & 0.26008 & $+g_{4}$ & 0.25944 \\
& & & & $+g_{5}$ & 0.25983 \\
\hline & $9 \times 10^{-4}$ & & $7 \times 10^{-4}$ & & $3 \times 10^{-4}$ \\
\hline
\end{tabular}

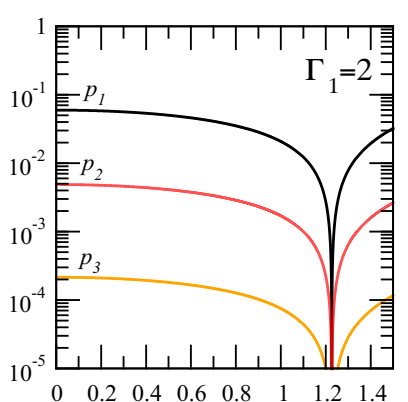

$\widetilde{\omega}$

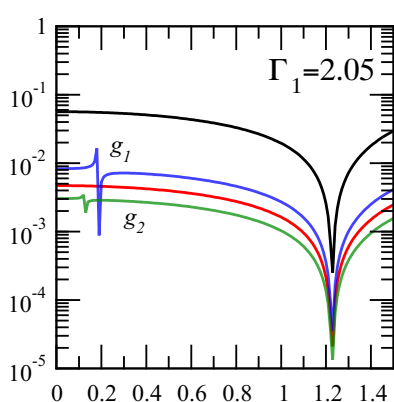

$\widetilde{\omega}$

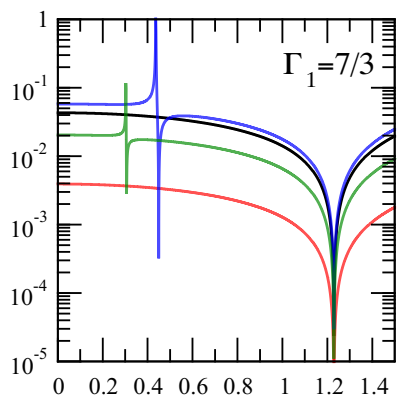

$\widetilde{\omega}$

Figure 1. Relative contributions to the tidal deformability (compared to that of the $f$-mode alone). The three panels show, from left to right: the barotropic case $\Gamma_{1}=\Gamma=2, \Gamma_{1}=2.05$ and $\Gamma_{1}=7 / 3$. Individual modes are colour coded (as indicated in the panels), with the same colour representing the same mode in all panels.

for the Love number. It is important to note that this is true also for the stratified models. The $f$-mode provides the dominant contribution in all cases (as expected given that this mode most closely resembles the tidal driving force), but in order to have a precise representation we need to account for both $p$-modes (pressure modes) and $g$-modes.

Having demonstrated that the sum over the star's oscillation modes provides a precise description of the tidal response in the static limit, let us turn to the dynamical response associated with finite frequencies, given by Eq. (6). It provides a closed expression for the frequency dependent tidal response (encoded in $k_{l}$ ). This allows us to quantify the level at which each individual mode contributes to the overall result. Results for the three different values of $\Gamma_{1}$ we have considered are presented in Fig. 1. The different panels show the relative contributions to the tidal deformability (compared to that of the $f$-mode alone). The resonances associated with each mode, which occur when $\tilde{\omega}=\tilde{\omega}_{n}$, are easily distinguishable 

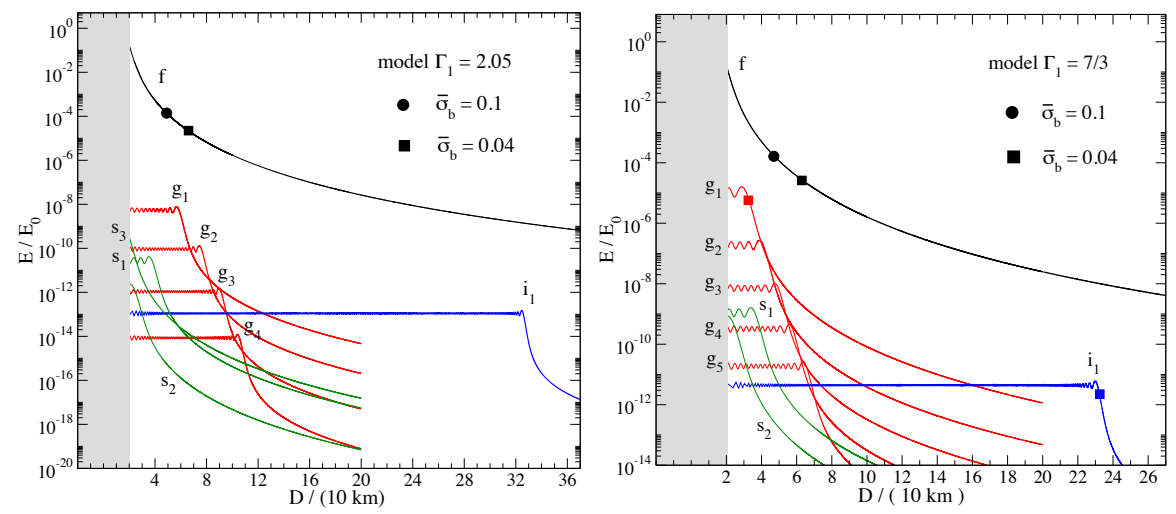

Figure 2. The evolution of the mode energies for varying binary separation. The energy is given in units of $E_{0}=G M_{\star}^{2} / R$. The model with $\Gamma_{1}=2.05$ is shown in the left-hand panel, while the $\Gamma_{1}=7 / 3$ case is provided in the right-hand panel. The energy of a mode increases when it becomes resonant during the inspiral. The breaking energy limit $E_{b}$ of the crust is represented by a circle or a square (see legend), which, respectively, denote the breaking energy for two choices of the crust breaking strain.

in each case and the resonance associated with the $f$-mode leads to a common feature in all panels. The results tell us that modes other than the $f$-mode contribute to the overall result at the few percent level. These results are important as they provide the first demonstration of the level at which frozen matter composition impacts on the tidal response across the range of frequencies relevant for a binary inspiral.

\section{Adding the crust}

When we turn to dynamical features of the tide, we need to be mindful of the fact that a neutron star interior is a little bit more complicated than a prescribed pressure-density relation. Nuclei in the lower density region are expected to freeze to form the so-called crust. This is important because the tidal response can be expressed as a sum over the stellar oscillation modes and the additional features of the interior physics may bring new modes into play and shift existing ones.

With this in mind, we studied the tidal response of a binary neutron star in models including an elastic crust and a fluid ocean. We showed that the presence of the crust does not significantly affect the fundamental, pressure, and buoyancy modes. As expected, the contribution to the Love number from the (crustal) shear modes is negligible, while the interface and surface $g$-modes have an impact similar to the first core $g$-modes. The influence of these modes, albeit small compared to the $f$-mode, increases for strongly stratified models.

Oscillations may be amplified by tidal resonances during the binary inspiral. This amplification is not only important for the gravitational-wave signal, but also for the impact that mode resonances can have on the crust. Thus, we studied the dynamical tidal evolution and determined the mode energy during the orbital shrinking. Our results confirm that the fundamental mode dominates the dynamical tides even when it is far from resonance. However, the interface modes are resonantly excited at an earlier stage and may accumulate enough energy to fracture the crust. This is mainly due to their peaked radial displacement at the crust-core or crust-ocean surface transition. The fundamental mode reaches the crust breaking limit in all our models, but not until the final part of the inspiral. Finally, we showed that the first 
$g$-mode can fracture the crust only for strongly stratified stars and (again) in the very final phase of inspiral. These results are shown in Fig. 2.

\section{A phenomenological model}

Based on the formalism presented in Sec. 2, we also developed a "phenomenological" model describing the dynamical tides in neutron star binaries. Our results demonstrate that the dynamical contribution to the tide is dominated by the excitation of the $f$-mode of the star, something that was established a long time ago [10] in work aimed at quantifying the impact of mode resonances on the gravitational-wave signal. Based on this, the basic idea is to include only the $f$-mode contribution to the mode sum and accept the contribution from other modes as a "systematic error." Based on the stratified Newtonian models considered before, we expect this error to be below the $5 \%$ level.

After doing this, the relation for the effective Love number that we end up with is

$$
k_{l}^{\mathrm{eff}} \approx \frac{\bar{\omega}_{f}^{2} k_{l}}{\bar{\omega}_{f}^{2}-\delta(2 \bar{\Omega})^{2}}+\frac{(2 \bar{\Omega})^{2}}{\bar{\omega}_{f}^{2}-\delta(2 \bar{\Omega})^{2}}\left[\frac{\delta}{2}-\frac{\bar{\omega}_{f}^{2}}{C^{3}} \frac{\epsilon}{l}\left(k_{l}+\frac{1}{2}\right)\right] .
$$

Here, the $f$-mode frequency is normalised as $\bar{\omega}_{f} \equiv M_{\star} \omega_{f}$ and can be obtained by a wellknown "universal relation" in terms of the (static) tidal deformability $k_{l}$ [20]. The orbital frequency is denoted by $\bar{\Omega}$ and the stellar compacness by $C$, which may also be expressed in terms of $k_{l}$ via another universal relation [21]. The factor $\epsilon / l$ represents the ratio $\left(V_{f} / W_{f}\right)_{R}$, with $\epsilon=1$ in a homogeneous star (and $\epsilon \approx 0.9$ for the polytropic models considered in Sec. 2). Finally, the parameter $\delta$ accounts for two relativistic effects: the gravitational redshift of the mode frequencies and the rotational frame-dragging induced by the orbital motion. An argument by Ref. [14] suggests that the two effects almost cancel, so-as a first approximation - we are motivated to simply remove the gravitational redshift altogether, implying that we may take $\delta=1-2 C$. Hence, we now have an explicit analytic formula for the effective Love number in terms of the result in the static limit, $k_{l}$, the orbital frequency $\bar{\Omega}$ and the (to some extent) free parameter $\epsilon$.

As a suitable comparison (in fact, the only comparable results in the literature), we consider the results for the dynamical tide from Refs. [13, 14], which are similar in spirit as they introduce the notion of an effective tidal deformability. However, the main focus of Refs. $[13,14]$ was to extend the effective-one-body framework to account for the dynamical tide. In addition to this, Ref. [14] provides an approximate analytical formula from matched asymptotics, which has been tested against numerical relativity simulations. As it appears to perform well in such comparisons, it provides a natural benchmark against which to test our simple closed-form expression.

Focussing on the example illustrated by Ref. [14], i.e., an equal mass neutron star binary with $M_{\star}=1.35 M_{\odot}$ and $R=13.5 \mathrm{~km}$, it is easy to demonstrate that we obtain an accurate representation of the dynamical tide throughout the relevant frequency range (up to close to merger) by tuning the parameter $\epsilon$. The effective Love number obtained from Eq. (10) is compared to the results from Ref. [14] in Fig. 3, for both $l=2$ and $l=3$. As our formula still leaves $\epsilon$ as a free parameter, we show results for the range $\epsilon=0.85-0.9$ and, as is clear from the result in the figure, the corresponding curves for $\epsilon=0.875$ provide an excellent match to the results from Ref. [14]. The essence of the comparison is that our formula (10) performs (perhaps surprisingly) well. It may be phenomenological in origin, but there can be little doubt that our simple expression provides an effective representation of the required behaviour, and hence reflects the underlying physics. 


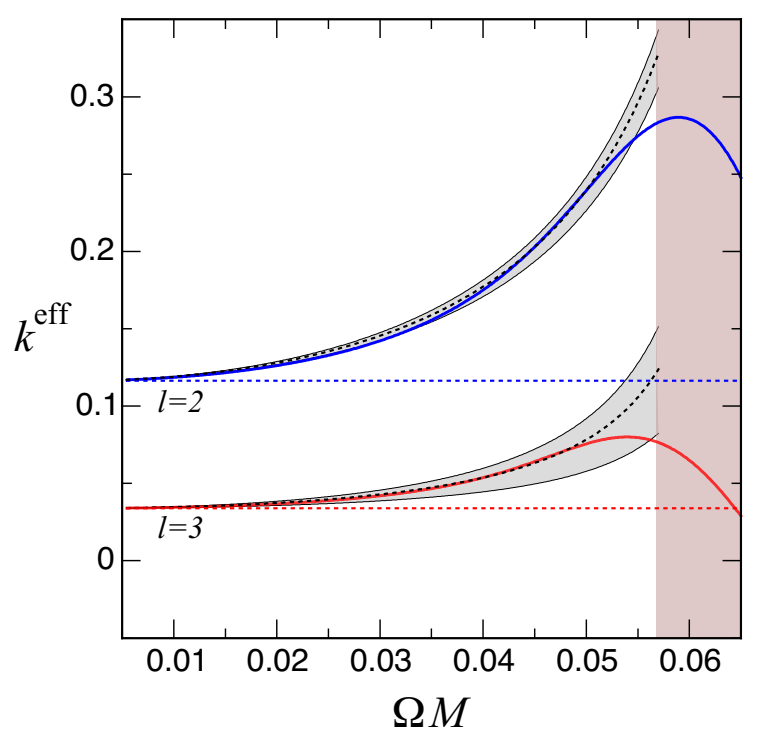

Figure 3. Comparing the effective Love number $k_{l}^{\text {eff }}$ from Eq. (10) with $\delta=1-2 C$ to the results from Ref. [14], for both $l=2$ and $l=3$. The dashed horizontal lines represent the static Love number $\left(k_{l}^{\text {eff }}\right.$ in the $\Omega \rightarrow 0$ limit). The results of Ref. [14] are shown as solid curves (blue for $l=2$ and red for $l=3$ ). Estimates from Eq. (10) are shown for the range $\epsilon=0.85-0.9$, with the latter representing the lower edge of the filled band in each case. The particular choice $\epsilon=0.875$ (dashed black curves) provides an an excellent fit to the results from Ref. [14]. Finally, we indicate the region beyond the (approximate) merger frequency, $\Omega M \gtrsim 0.057$ in this case, by the shaded area in the figure.

It is worth noting that, while the two sets of results diverge for large values of $\Omega M$ in Fig. 3 (where $M=M_{\star}+M^{\prime}$ ), the corresponding frequencies are close to (or indeed beyond) the merger frequency. As the picture of two separate, tidally deformed, bodies breaks down there is no reason to expect the model to make sense beyond this point. The post-merger region is indicated by the shaded area in Fig. 3.

\section{Implications}

We have discussed the tidal response of a neutron star during the late stages of neutron star binary inspiral. In particular, we have focussed on the role of the matter composition. This issue has previously been ignored as studies have almost exclusively focussed of barotropic fluid models. However, it is natural to argue (given the time scale involved) that the matter composition should remain "frozen" during the late stages of binary inspiral, leading to a stratified perturbation problem (where the adiabatic index of the perturbation is different from that of the equilibrium background). This connects with previous work on tidal resonances, which has quantified the role of the $g$-modes (which rely on stratification for their existence).

Our numerical results indicate that the difference is at (or below) the level of a few percent. However, it is nevertheless important to quantify this contribution. We need to do this in order to understand systematic "errors" associated with the assumed physics, which ultimately determines the accuracy with which we can hope to extract stellar parameters like the radius from observations. Today's gravitational-wave detectors are not at a level where a 
change of a percent in the tidal response makes much difference, but one might want to keep an eye on these issues for future reference.

The problem is also important from the physics point of view. Having quantified the level at which the matter composition enters the discussion, we can compare to the role of other elements of neutron star physics, like an elastic crust. Moreover, this formalism allowed us to develop a phenomenological, physically motivated, model for the effective tidal deformability of a neutron star binary, adding frequency dependence that comes into play during the late stages of inspiral. A comparison against alternative descriptions suggests that we have at hand a simple, yet accurate, description of the tidal imprint. This should make the model an attractive alternative for an implementation of the matter effects in gravitational-wave data analysis algorithms.

P.P. and N.A. acknowledge support from the Science and Technology Facilities Council (STFC) via grant ST/R00045X/1. P.P. acknowledges support from the "Ministero dell'istruzione, dell'università e della ricerca" (MIUR) PRIN 2017 programme (CUP: B88D19001440001) and from the Amaldi Research Center funded by the MIUR programme "Dipartimento di Eccellenza" (CUP: B81I18001170001).

\section{References}

[1] B.P. Abbott et al., Phys. Rev. Lett. 119, 161101 (2017), 1710.05832

[2] B.P. Abbott et al., Astrophys. J. 892, L3 (2020), 2001.01761

[3] É.É. Flanagan, T. Hinderer, Phys. Rev. D 77, 021502 (2008), 0709. 1915

[4] T. Hinderer, B.D. Lackey, R.N. Lang, J.S. Read, Phys. Rev. D 81, 123016 (2010), 0911. 3535

[5] B.P. Abbott et al., Classical Quantum Gravity 35, 065009 (2018), 1711.06843

[6] M.C. Miller et al., Astrophys. J. 887, L24 (2019), 1912 . 05705

[7] T.E. Riley et al., Astrophys. J. 887, L21 (2019), 1912 . 05702

[8] M.C. Miller et al., Astrophys. J. 918, L28 (2021), 2105.06979

[9] T.E. Riley et al., Astrophys. J. 918, L27 (2021), 2105 . 06980

[10] D. Lai, Mon. Not. R. Astron. Soc. 270, 611 (1994), astro-ph/9404062

[11] A. Reisenegger, P. Goldreich, Astrophys. J. 426, 688 (1994)

[12] K.D. Kokkotas, G. Schäfer, Mon. Not. R. Astron. Soc. 275, 301 (1995), gr-qc/9502034

[13] T. Hinderer et al., Phys. Rev. Lett. 116, 181101 (2016), 1602.00599

[14] J. Steinhoff, T. Hinderer, A. Buonanno, A. Taracchini, Phys. Rev. D 94, 104028 (2016), 1608.01907

[15] N. Andersson, P. Pnigouras, Mon. Not. R. Astron. Soc. 503, 533 (2021), 1905.00012

[16] P. Schmidt, T. Hinderer, Phys. Rev. D 100, 021501 (2019), 1905.00818

[17] G. Pratten, P. Schmidt, N. Williams (2021), 2109.07566

[18] N. Andersson, P. Pnigouras, Phys. Rev. D 101, 083001 (2020), 1906.08982

[19] A. Passamonti, N. Andersson, P. Pnigouras, Mon. Not. R. Astron. Soc. 504, 1273 (2021), 2012.09637

[20] T.K. Chan, Y.H. Sham, P.T. Leung, L.M. Lin, Phys. Rev. D 90, 124023 (2014), 1408. 3789

[21] A. Maselli, V. Cardoso, V. Ferrari, L. Gualtieri, P. Pani, Phys. Rev. D 88, 023007 (2013), 1304.2052 